\title{
Comparison of Epworth Sleepiness Scale and STOP-BANG Questionnaire for Diagnosing Obstructive Sleep Apnea at a Tertiary Care Centre in North India: A Retrospective Study
}

\author{
Shibdas Chakrabarti ${ }^{1}$, Mansunderbir Singh ${ }^{2}$, Rohit Kumar ${ }^{3}$, Nitesh Gupta ${ }^{4}$, Vidushi Rathi ${ }^{5}$, Pranav Ish ${ }^{6}$
}

\begin{abstract}
Introduction: The diagnosis of obstructive sleep apnea (OSA) requires polysomnography (PSG). The screening of OSA utilizes questionnaires like the Epworth sleepiness scale (ESS) and the STOP-BANG questionnaire (SBQ) that have variable predictive value, as reported from the Western literature.

Materials and methods: A retrospective cohort study was done in adult subjects $(n=80)$ presenting with symptoms of OSA. The demographic profile, ESS, and SBQ scores were evaluated. The level one recorded PSG was evaluated for apnea-hypopnea index (AHI) and positive airway pressure (PAP) therapy prescribed.

Results: Of the 80 subjects, 75 were diagnosed as OSA on PSG. The mean age of the study group was 49 years, with $71 \%$ men and a mean BMI of $28.25 \mathrm{~kg} / \mathrm{m}^{2}$. Epworth sleepiness scale $\geq 11$ was in $62.66 \%$, and SBQ $\geq 5$ in $53 \%$ of the 75 subjects. Hence, both questionnaires failed to predict OSA in nearly half of the population. The mean AHI was 33.8/hour, and the mean continuous positive airway pressure (CPAP) was $10.05 \mathrm{~cm}$ $\mathrm{H}_{2} \mathrm{O}$. The AHI had a significant correlation with BMI, ESS score, and CPAP. Epworth sleepiness scale had a $53 \%$ sensitivity and $60 \%$ specificity for diagnosing OSA using a cutoff of 11, whereas SBQ had a $68 \%$ sensitivity and $100 \%$ specificity using a cutoff of 5 .

Conclusion: The SBQ has a higher sensitivity and specificity to detect OSA than ESS as it envisages distinct clinical manifestations and risk factors of OSA. However, neither of the two can replace PSG.
\end{abstract}

Keywords: Epworth sleepiness scale, Obstructive sleep apnea, Polysomnography, Screening, STOP-BANG questionnaire.

Indian Journal of Sleep Medicine (2019): 10.5005/jp-journals-10069-0042

\section{INTRODUCTION}

Obstructive sleep apnea (OSA) is a common disorder characterized by snoring, daytime sleepiness, fatigue, repeated termination of airflow (apnea), and hypoxemia. ${ }^{1}$ It is an independent risk factor for cardiovascular diseases including hypertension, coronary artery disease, stroke, and pulmonary hypertension. The gold standard diagnostic technique is polysomnography (PSG), ${ }^{2}$ which is a noninvasive technique that involves overnight monitoring of physiological variables including electroencephalography, eye movements, muscle tone, respiratory effort, airflow, and oxygen saturation. The severity of OSA is graded according to commonly used clinical criteria as mild ( $\mathrm{AHI} \geq 5$ but $<15$ ), moderate $(\mathrm{AHI} \geq 15$ but $<30$ ), or severe $(\mathrm{AHI} \geq 30){ }^{3}$ Acknowledging the high cost of and inaccessibility to PSG, several screening questionnaires were formulated. The most conventional questionnaires are the STOPBANG questionnaire and ESS. These questionnaires have a wide range of sensitivity and specificity in different races. ${ }^{4-7}$ Hence, we conducted this study to compare the predictive values of these questionnaires in the North Indian population.

\section{Lacunae in Existing Knowledge}

The studies comparing two questionnaires (SBQ and ESS) are limited to the Western population. The comparison is often unsupported by level 1 PSG, and hence real reliability is unknown.

\section{Aims and Objectives}

- To compare the two questionnaires (SBQ and ESS) for detecting OSA

- To correlate AHI with SBQ, ESS, and BMI.
${ }^{1-6}$ Department of Pulmonary, Critical Care and Sleep Medicine, Vardhman Mahavir Medical College and Safdarjung Hospital, New Delhi, India

Corresponding Author: Pranav Ish, Department of Pulmonary, Critical Care and Sleep Medicine, Vardhman Mahavir Medical College and Safdarjung Hospital, New Delhi, India, Phone: +91 9958356000, e-mail: pranavish2512@gmail.com

How to cite this article: Chakrabarti $S$, Singh $M$, Kumar $R$, et al. Comparison of Epworth Sleepiness Scale and STOP-BANG Questionnaire for Diagnosing Obstructive Sleep Apnea at a Tertiary Care Centre in North India: A Retrospective Study. Indian J Sleep Med 2019;14(3):46-50.

Source of support: Nil

Conflict of interest: None

\section{Materials and Methods}

The current study is a retrospective cohort study. Adult subjects who visited the sleep clinic between October 1, 2018, and March 3, 2019 with suspected OSA were included.

\section{Inclusion Criteria}

- Age above 18 years.

- Obstructive sleep apnea symptoms (fatigue or sleepiness during the day, nocturnal snoring or choking, and observed apnea by others), no previous treatment, completion of questionnaires, and personal consent to do PSG and participate in the study.

o The Author(s). 2019 Open Access This article is distributed under the terms of the Creative Commons Attribution 4.0 International License (https://creativecommons. org/licenses/by-nc/4.0/), which permits unrestricted use, distribution, and non-commercial reproduction in any medium, provided you give appropriate credit to the original author(s) and the source, provide a link to the Creative Commons license, and indicate if changes were made. The Creative Commons Public Domain Dedication waiver (http://creativecommons.org/publicdomain/zero/1.0/) applies to the data made available in this article, unless otherwise stated. 


\section{Exclusion Criteria}

- Subjects with incompletely finished questionnaire

- A history of psychiatric disorder.

All subjects had filled SBQ and ESS questionnaires and undergone a PSG in the time frame. A detailed history to rule out other causes of excessive daytime sleepiness including occupational working hours, depression, other psychiatric disorders, symptoms suggestive of hypothyroidism, sedative drug use, etc. was taken.

The SBQ questionnaire includes four subjective (STOP: snoring, tiredness, observed apnea, and high blood pressure) and four demographics items [BANG: body mass index (BMI), age, neck circumference, and gender]. Answering "yes" to five or more items is categorized as high risk for the OSA. ${ }^{8}$ STOP-BANG questionnaire is validated in the meta-analysis for the screening of OSA in a sleep clinic and surgical population. The ESS is an eight-item questionnaire to measure daytime sleepiness. The questionnaire has a four-point Likert response format (0-3), and the score ranges from 0 to 24 . Epworth sleepiness scale score $\geq 11$ indicates excessive daytime sleepiness and high risk for OSA. ${ }^{8}$

Standard attended overnight PSG (Philips Respironics) was done in these subjects in the defined time frame. Electroencephalogram, electrocardiogram, electrooculogram, chin electromyogram, oronasal airflow, oxygen saturation by pulse oximetry, and thoracic and abdominal movements were recorded. Scoring of events were done according to the 2015 American Association of Sleep Medicine guidelines. ${ }^{9}$ Subjects were classified based on the $A H I$ values without OSA $(\mathrm{AHI}<5)$, mild OSA $(5 \leq \mathrm{AHI}<15)$, moderate OSA (15 $\leq \mathrm{AHI}<30)$, and severe OSA $(\mathrm{AHI} \geq 30)$. The clinician, blinded to the results of questionnaires, interpreted the $\mathrm{AHI}$ independently.

\section{Statistical Analysis}

SPSS, version 17.0 (SPSS Science, Apache Software Foundation, Chicago, IL, USA), was used for data analysis. The Pearson's correlation coefficient was used for the questionnaires in subjects. $p<0.05$ was accepted as statistically significant.

\section{Results}

A total of 80 subjects recorded ESS and SBQ questionnaire. The previous history of comorbidities, including hypertension, diabetes and coronary artery disease was documented. The PSG results of subjects were analyzed for apnea-hypopnea index and titration pressures.

Of the 80 subjects, 75 were diagnosed as having OSA on PSG. The mean age of the study group was 49 years, with $71 \%$ men and a mean BMI of $28.25 \mathrm{~kg} / \mathrm{m}^{2}$. Epworth sleepiness scale $\geq 11$ was in $62.66 \%$, and SBQ $\geq 5$ in $53 \%$ of the 75 subjects. Hence, both questionnaires failed to predict OSA in nearly half of the population. The metabolic comorbidities were common in population with OSA: $40 \%$ had hypertension, $32 \%$ had diabetes, and nearly $12 \%$ had a history of coronary artery disease.

The mean AHI was 33.8/hour, and the mean CPAP was 10.05 $\mathrm{Cm} \mathrm{H}_{2} \mathrm{O}$ (Figs 1 to 4). The mean values of arterial blood gas analysis and spirometry were in the normal range (Table 1).

Using the Pearson's correlation coefficient, $\mathrm{AHI}$ had a significant correlation with BMI, ESS score, and CPAP titrated, but surprisingly not with SBQ. Epworth sleepiness scale score and SBQ did have a significant correlation with each other (Table 2). Epworth sleepiness scale had $53 \%$ sensitivity and $60 \%$ specificity for diagnosing OSA using a cutoff of 13 , whereas SBQ had $68 \%$ sensitivity and $100 \%$ specificity using a cutoff of 5 .

\section{Discussion}

The current study is a reflection of the demographic profile of OSA at a tertiary care center in North India. The disease peaks at 40-60 years of age, is more prevalent in men, and is associated with metabolic comorbidities like hypertension and diabetes mellitus.

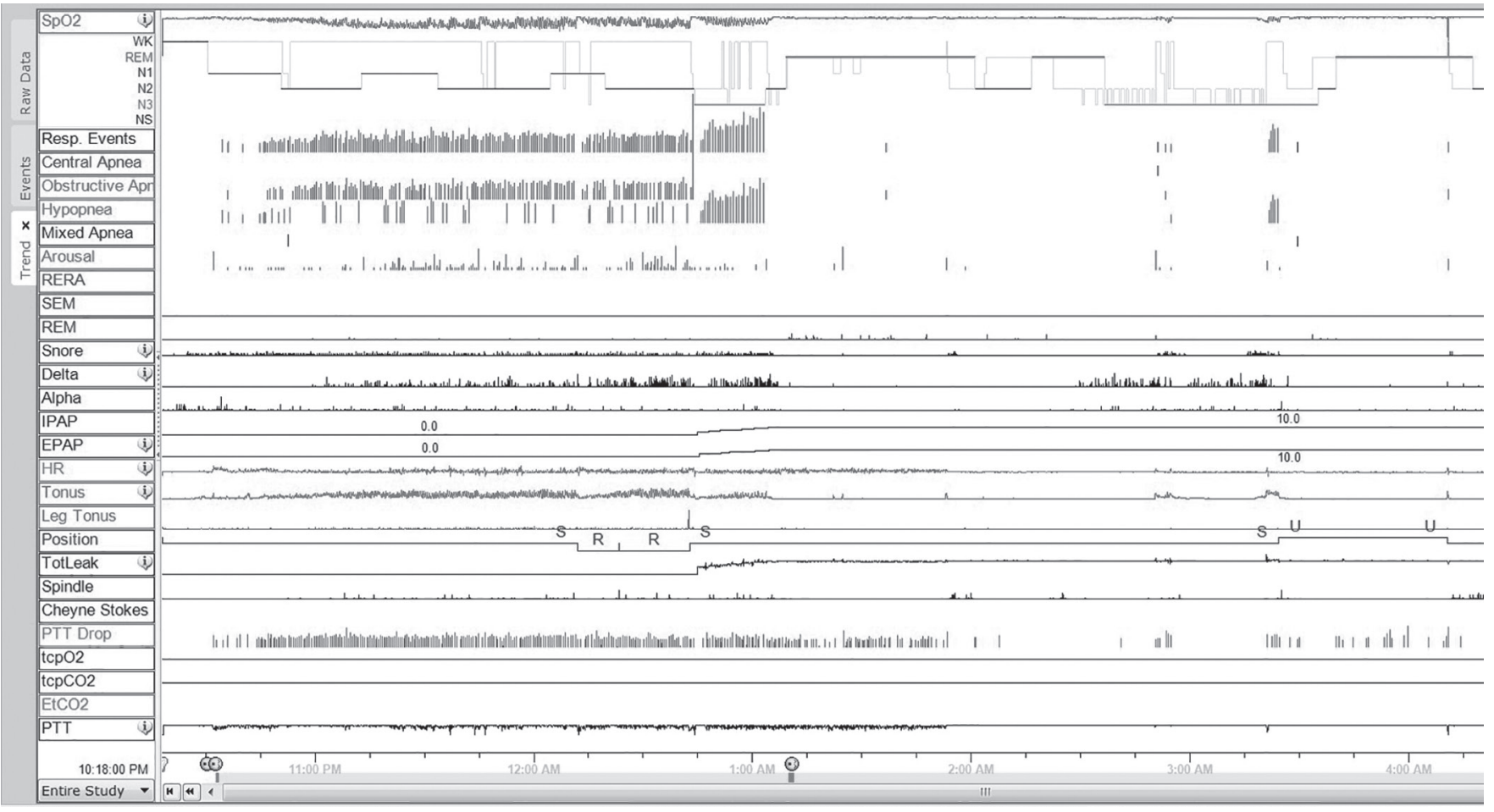

Fig. 1: Hypnogram showing severe obstructive sleep apnea titrated with CPAP of $10 \mathrm{~cm} \mathrm{H}_{2} \mathrm{O}$ 
Comparison of ESS and STOP-BANG Questionnaire for diagnosing OSA at a Tertiary Care Centre in North India

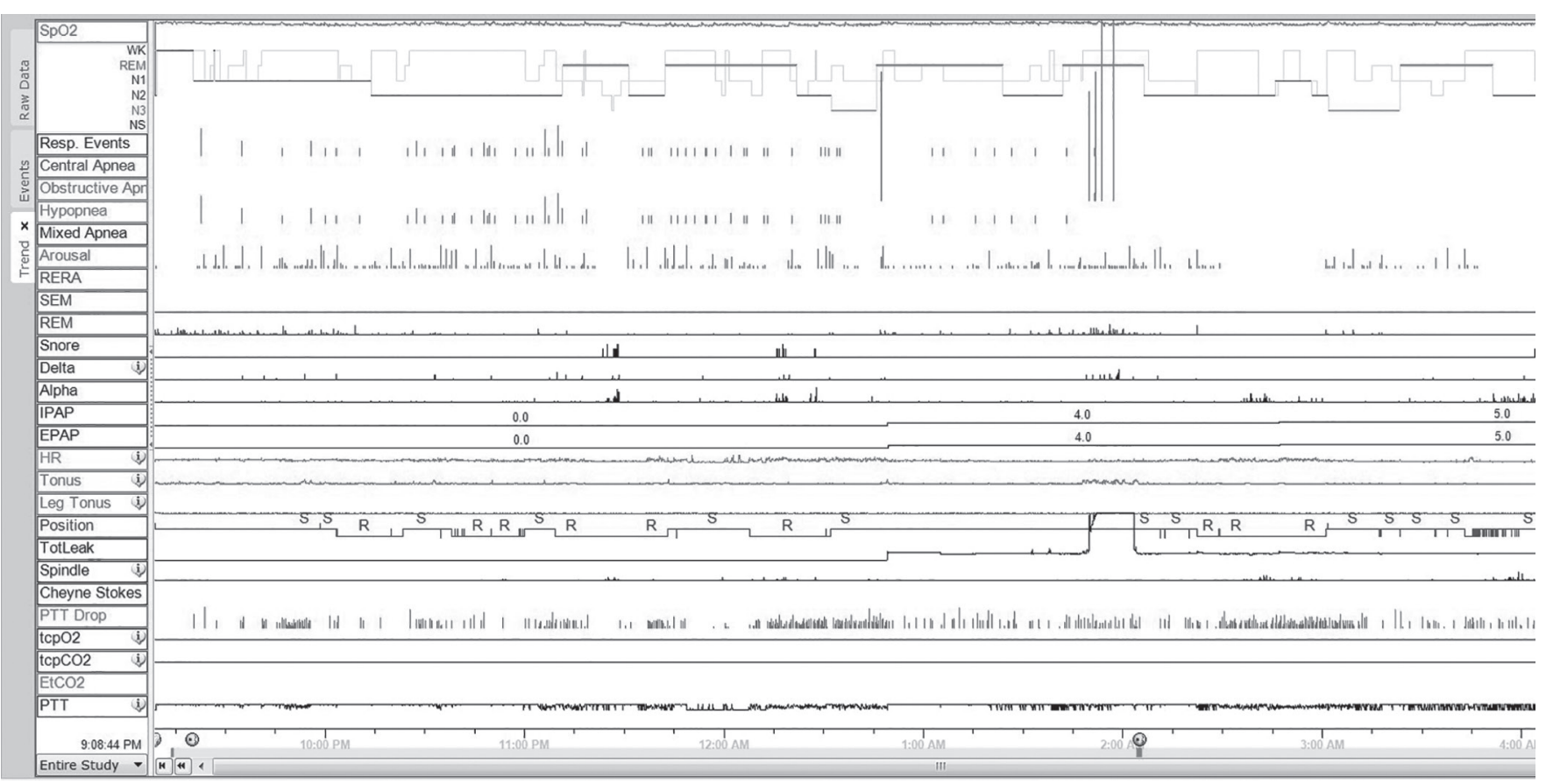

Fig. 2: Hypnogram showing moderate obstructive sleep apnea titrated with CPAP of $5 \mathrm{~cm} \mathrm{H}_{2} \mathrm{O}$

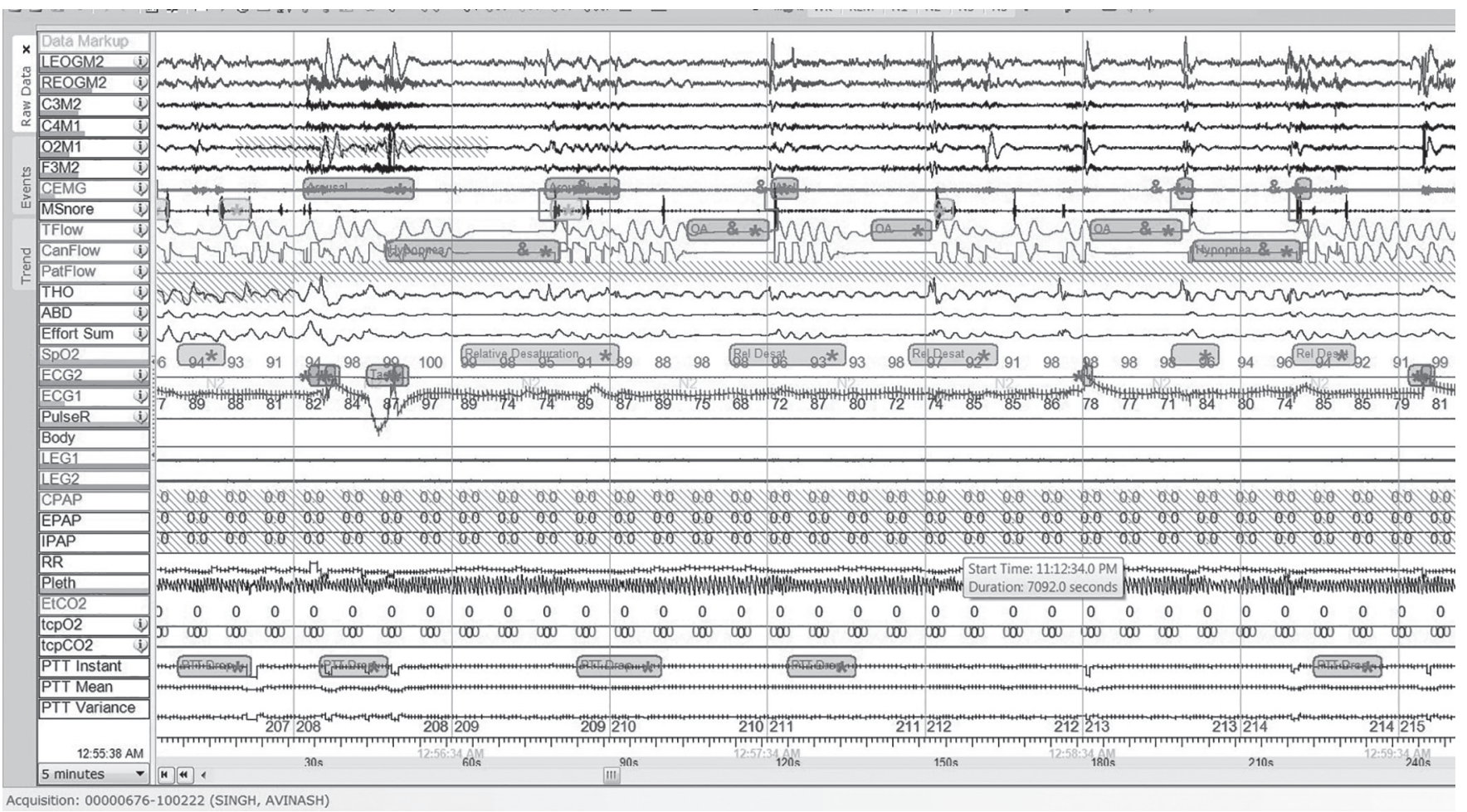

Fig. 3: A 5-minute window of polysomnogram showing 10 epochs with numerous obstructive apneas and hypopneas

Diagnosing OSA is a big dilemma given the lack of awareness among both subjects and doctors, lack of standard questionnaire, and the ultimate need to carry out a PSG, both for diagnosis and for titration. The facility of PSG, even in urban parts of India, is limited, though emerging. Interpretation of these studies is equally tricky and time-consuming. Hence there is an urgent need for highly sensitive and specific questionnaires to predict OSA so that the appropriate target population undergoes the required PSG and titration.

In the present study, SBQ was found to be more sensitive as well as specific than ESS for OSA in predicting the diagnosis of OSA. The studies in the past have given conflicting evidence for the same. A study from western India analyzed 50 patients and found ESS to have higher sensitivity and negative predictive value than SBQ 
Comparison of ESS and STOP-BANG Questionnaire for diagnosing OSA at a Tertiary Care Centre in North India

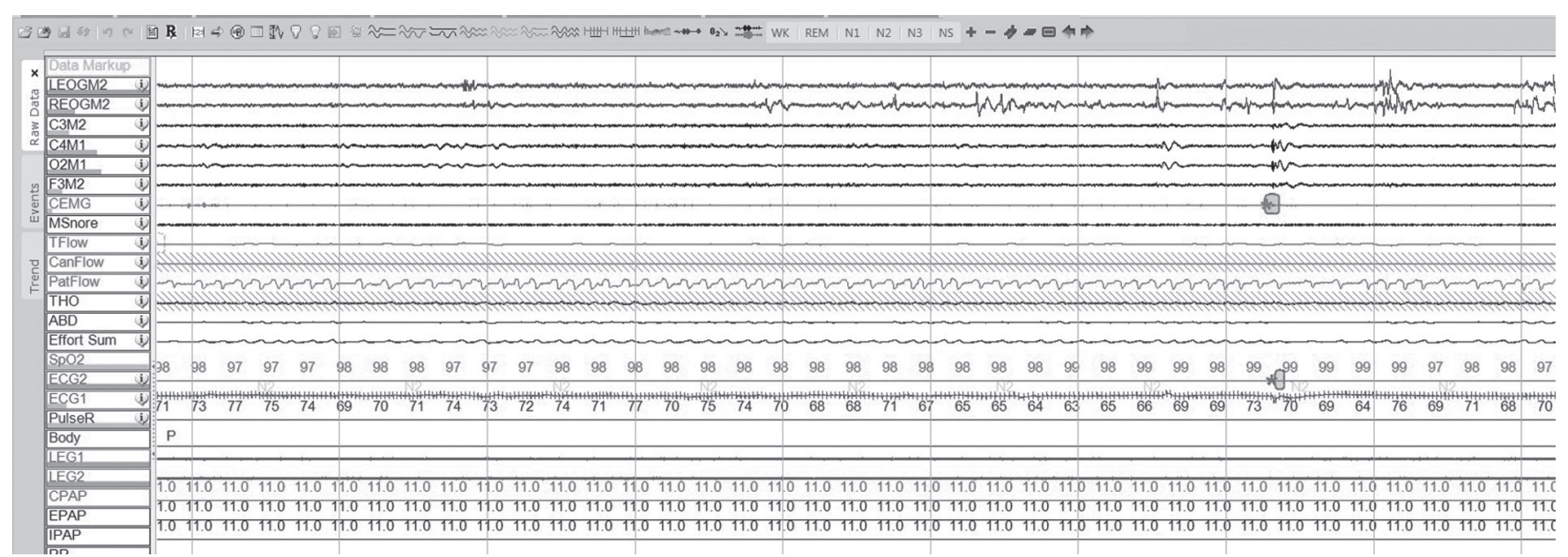

Fig. 4: A 5-minute window of polysomnogram showing resolution of the apneas and hypopneas with CPAP of $11 \mathrm{~cm} \mathrm{H}_{2} \mathrm{O}$

Table 1: Descriptive statistics of the study population

\begin{tabular}{llc}
\hline Parameter & Mean & SD \\
\hline Age & 49.10 & 11.692 \\
$\mathrm{BMI}$ & 28.256 & 5.1559 \\
$\mathrm{ESS}$ & 12.40 & 5.274 \\
$\mathrm{SBQ}$ & 4.80 & 1.444 \\
$\mathrm{PH}$ & 7.416 & 0.0227 \\
$\mathrm{pO}_{2}$ & 77.79 & 11.339 \\
$\mathrm{pCO}_{2}$ & 38.390 & 7.9267 \\
$\mathrm{HCO}_{3}$ & 24.101 & 2.5760 \\
$\mathrm{FEV} 1$ & 85.16 & 18.281 \\
$\mathrm{FVC}$ & 88.15 & 17.220 \\
FEV1/FVC ratio & 79.04 & 9.808 \\
AHI & 33.843 & 23.4351 \\
CPAP & 10.05 & 3.778 \\
\hline AHI & &
\end{tabular}

$\mathrm{AHI}$, apnea-hypopnea index; BMI, body mass index; ESS, Epworth sleepiness score; SBQ, STOP-BANG questionnaire; CPAP, continuous positive airway pressure; $\mathrm{pO}_{2}$, partial pressure of oxygen in blood; $\mathrm{pCO}_{2}$, partial pressure of carbon dioxide in blood; $\mathrm{HCO}_{3}$, serum bicarbonate; $\mathrm{FEV} 1$, forced expiratory volume in 1 second; FVC, forced vital capacity; SD, standard deviation

Table 2: Correlation between apnea hypopnea index and body mass index, Epworth sleepiness score, STOP-BANG questionnaire and continuous positive airway pressure

\begin{tabular}{llllll}
\hline Correlation & & BMI & ESS & SBQ & CPAP \\
\hline AHI & $\begin{array}{l}\text { Pearson } \\
\text { correlation }\end{array}$ & $0.509^{* *}$ & $0.301^{* *}$ & 0.219 & $0.451^{* *}$ \\
& & & & \\
\hline
\end{tabular}

** Correlation is significant at the 0.01 level (2-tailed)

AHI, apnea-hypopnea index; BMI, body mass index; ESS, Epworth sleepiness score; SBQ, STOP-BANG questionnaire; EPAP, expiratory positive airway pressure

in detecting OSA. ${ }^{10}$ However, most studies agree that SBQ has a higher sensitivity toward diagnosing OSA. Comparing ESS to other questionnaires like the Berlin questionnaire, it fared poorly both in sensitivity and in specificity. ${ }^{11}$

This study also shows that ESS and SBQ can miss nearly half of the cases for diagnosing OSA. The reasons can be many: OSA is a multietiology disorder with a myriad of risk factors, symptoms, and treatment options. Phenotyping OSA according to the age of presentation helps in deciding the treatment options ranging from tonsillectomy, mandibular advancement devices, and PAP therapy. ${ }^{11}$ Adolescents and children frequently do not have symptoms of excessive daytime sleepiness despite having severe OSA. Such children have the clinical phenotype of "mouth breathing" or "isolated poor neurocognitive development." Timely intervention in such patients with adenotonsillectomy, oral devices, and advancement surgeries can prevent future morbidity. Similarly, the elderly population frequently present with insomnia due to OSA. Such patients need PAP therapy to correct OSA and also to improve their sleep quality to a large extent. A recent case report highlights the side effects of hypnotics and sedatives taken for insomnia when the patient had underlying OSA. The ESS in the said patient was $2 .{ }^{12}$ Hence, questionnaires like ESS are bound to be of limited use in these patients. Thus, ESS can only be employed as a measure of the severity of excessive daytime sleepiness (EDS) and cannot be used as a tool to predict the probability of OSA due to the mentioned factors. STOP-BANG questionnaire is a more comprehensive screening tool as it includes symptoms, comorbidities, and physical examination findings of the patients, thus, faring better than ESS and found validated for screening patients, primarily surgical patients. ${ }^{8}$ However, studies have found SBQ to be less useful in veteran ${ }^{13}$ and chronic kidney disease patients. ${ }^{14}$ Most studies, which register SBQ having high sensitivity and specificity, have a selection bias or are not validated by in-lab (level one) PSG. ${ }^{8}$ In a study validating the results by a PSG, SBQ was found to have the best negative likelihood ratio among nine questionnaires in predicting the presence of OSA in over 200 subjects. ${ }^{15} \mathrm{~A}$ recent retrospective study concluded that perioperative sleep apnea prediction score (PSAP), SBQ, and ESS together, if negative, virtually rules out OSA. ${ }^{16}$ In the present study, all the subjects validated with a PSG, thereby proving the superior sensitivity and specificity of SBQ over ESS. A selection bias, however, cannot be ruled out as all these patients presented to a tertiary care clinic and were bound to be symptomatic, and most of them had severe OSA.

\section{Conclusion}

Screening for OSA by ESS and SBQ has many limitations. These questionnaires cannot replace PSG. However, SBQ has a higher sensitivity and specificity to detect OSA than ESS and may be used in controlled settings to prioritize patients for subsequent PSG for diagnosing OSA and PAP titration. The search for an ideal screening tool is in continuum. 


\section{Declaration of Patient Consent}

The authors certify that they have obtained all appropriate patient consent forms. The patients provided their consent for using the images and other clinical information to be reported in the journal.

The patients understand that their name and initials will not be published and due efforts will be made to conceal their identity, but anonymity cannot be guaranteed.

\section{References}

1. Amra B, Farajzadegan Z, Golshan M, et al. Prevalence of sleep apnea-related symptoms in a persian population. Sleep Breath 2011;15(3):425-429. DOI: 10.1007/s11325-010-0353-4.

2. Amra B, Nouranian E, Golshan M, et al. Validation of the persian version of berlin sleep questionnaire for diagnosing obstructive sleep apnea. Int J Prev Med 2013;4(3):334-339.

3. Pamidi S, Aronsohn RS, Tasali E. Obstructive sleep apnea: role in the risk and severity of diabetes. Best Pract Res Clin Endocrinol Metab 2010;24(5):703-715. DOI: 10.1016/j.beem.2010.08.009.

4. Chiu HY, Chen PY, Chuang LP, et al. Diagnostic accuracy of the berlin questionnaire, STOP-BANG, STOP, and Epworth sleepiness scale in detecting obstructive sleep apnea: a bivariate meta-analysis. Sleep Med Rev 2017;36:57-70. DOI: 10.1016/j.smrv.2016.10.004.

5. Nagappa M, Liao P, Wong J, et al. Validation of the STOP-bang questionnaire as a screening tool for obstructive sleep apnea among different populations: a systematic review and meta-analysis. PLoS One 2015;10(12):e0143697. DOI: 10.1371/journal.pone.0143697.

6. Nagappa M, Wong J, Singh M, et al. An update on the various practical applications of the STOP-bang questionnaire in anesthesia, surgery, and perioperative medicine. Curr Opin Anaesthesiol 2017;30:118-125. DOI: $10.1097 / A C O .0000000000000426$.

7. Sharma A, Molano J, Moseley BD. The STOP-BANG questionnaire improves the detection of epilepsy patients at risk for obstructive sleep apnea. Epilepsy Res 2017;129:37-40. DOI: 10.1016/j.eplepsyres. 2016.11.009.

8. Chung F, Yegneswaran B, Liao P, et al. STOP questionnaire: a tool to screen patients for obstructive sleep apnea. Anesthesiology 2008;108(5):812-821. DOI: 10.1097/ALN.0b013e31816d83e4.

9. Berry RB, Gamaldo CE, Harding SM, et al. AASM scoring manual version 2.2 updates: new chapters for scoring infant sleep staging and home sleep apnea testing. J Clin Sleep Med 2015;11(11):1253-1254. DOI: 10.5664/jcsm.5176.

10. Dixit R, Verma S, Pawar KS. Screening for obstructive sleep apnea using epworth sleepiness score and berlin questionnaire: Which is better? Indian J Respir Care 2018;7:33-36. DOI: 10.4103/ijrc.ijrc_20_17.

11. Mittal A, Suri JC, Chakrabarti S, et al. Therapeutic cure of obesity hypoventilation syndrome resulting in cure of obstructive sleep apnea and metabolic syndrome. Indian Sleep Med 2019;14(1):18-21. DOI: 10.5005/jp-journals-10069-0034.

12. Chakrabarti S, Darisetty S, Gupta N, et al. Masking of obstructive sleep apnoea by drug induced central sleep apnoea - a case report. Adv Respir Med 2019;87(3):189-193. DOI: 10.5603/ARM.2019.0030.

13. Kunisaki KM, Brown KE, Fabbrini AE, et al. STOP-BANG questionnaire performance in a veterans affairs unattended sleep study program. Ann Am Thorac Soc 2014;11(2):192-197. DOI: 10.1513/ AnnalsATS.201305-134OC.

14. Nicholl DD, Ahmed SB, Loewen AH, et al. Diagnostic value of screening instruments for identifying obstructive sleep apnea in kidney failure. J Clin Sleep Med 2013;9(1):31-38. DOI: 10.5664/jcsm.2334.

15. Prasad KT, Sehgal IS, Agarwal R, et al. Assessing the likelihood of obstructive sleep apnea: a comparison of ninescreening questionnaires. Sleep Breath 2017;21(4):909-917. DOI: 10.1007/s11325017-1495-4.

16. Saxena M, Gothi D, Sah R, et al. Utility of combining Epworth sleepiness scale, stop-bang and perioperative sleep apnea prediction score for predicting absence of obstructive sleep apnea. Indian Sleep Med 2018;13(4):62-66. DOI: 10.5005/jp-journals-10069-0028. 